International Journal of Modern Physics B

Vol. 26, No. 26 (2012) 1292002 (1 page)

(C) World Scientific Publishing Company

DOI: $10.1142 /$ S0217979212920026

\title{
ERRATUM
}

\section{THE LUTTINGER LIQUID AND INTEGRABLE MODELS}

\author{
[Int. J. Mod. Phys. B, Vol. 26, No. 22 (2012) 1244009 (24 pages)] \\ J. SIRKER \\ Department of Physics and Research Center OPTIMAS, \\ Technical University Kaiserslautern, \\ D-67663 Kaiserslautern, Germany \\ Published 5 September 2012
}

Due to a misprint, the correct version for the lines below is as follows:

(1) On the second last line at the end of Chapter 2.1, p. 3, in the sentence "... can give rise to a finite bound in Eq. (7) so that $D(D>0) \neq 0 \ldots$. $D(D>0)$ should be $D(T>0)$ instead.

(2) The correct version of Eq. (65) is:

$$
\delta \chi_{2}^{\text {uni }}=\frac{\pi v b}{2 K T^{2} L^{3}}[\cdots]
$$

(i.e., " $a$ " in " $\pi v a "$ should be changed to " $b$ " instead; the part of the formula within the square brackets remains unchanged.) 